\title{
Cutaneous immune responses mediated by dendritic cells and mast cells
}

\author{
Tina L. Sumpter, ${ }^{1}$ Stephen C. Balmert, ${ }^{1}$ and Daniel H. Kaplan ${ }^{1,2}$ \\ 'Department of Dermatology and 'Department of Immunology, University of Pittsburgh School of Medicine, \\ Pittsburgh, Pennsylvania, USA.
}

In the skin, complex cellular networks maintain barrier function and immune homeostasis. Tightly regulated multicellular cascades are required to initiate innate and adaptive immune responses. Innate immune cells, particularly DCs and mast cells, are central to these networks. Early studies evaluated the function of these cells in isolation, but recent studies clearly demonstrate that cutaneous DCs (dermal DCs and Langerhans cells) physically interact with neighboring cells and are receptive to activation signals from surrounding cells, such as mast cells. These interactions amplify immune activation. In this review, we discuss the known functions of cutaneous DC populations and mast cells and recent studies highlighting their roles within cellular networks that determine cutaneous immune responses.

Conflict of interest: The authors have declared that no conflict of interest exists.

Published: January 10, 2019

\section{Reference information:}

JCI Insight. 2019;4(1):e123947. https://doi.org/10.1172/jici. insight.123947.

\section{Introduction}

In the skin, cellular networks maintain immune homeostasis and initiate protective innate and adaptive immune responses. DCs and mast cells are central within this network. Mast cells provide immediate innate immune signals to cells in the surrounding microenvironment. DCs, the most potent professional antigen-presenting cells, are essential for induction of adaptive immunity. However, the primary functions of DCs and mast cells are not achieved in isolation. Rather, initiation of innate and adaptive immune responses reflects intricate interactions between diverse cell types residing in the cutaneous microenvironment, including nonimmune cells such as keratinocytes and sensory nerve fibers.

The cells forming the immune barrier in the skin have long been investigated independently, but recent focus has shifted toward understanding how these cells interact in context with one another and how their interactions facilitate coordinated innate and adaptive immune responses. In this review, we describe recent findings illustrating the importance of cellular networks in the skin. We highlight discoveries defining the physical interactions between the highly specialized epidermal Langerhans cells and their neighboring keratinocytes in relationship to adaptive immunity. We then discuss cellular interactions in the dermis, with a focus on dermal DCs and mast cells. Last, we discuss recent findings investigating the impact of cellular interactions between DCs or mast cells and a new player in innate immune responses, sensory nerve fibers. Collectively, these studies support the view that cellular interactions are critical for initiation of innate immune responses and subsequent adaptive immune responses in the skin.

\section{Interactions between Langerhans cells and keratinocytes}

The skin is anatomically divided into epidermis and dermis in mice and into epidermis, dermis, and hypodermis in humans. Langerhans cells, the sole professional antigen-presenting cells of the epidermis, are embedded within the stratum of tightly linked keratinocytes. The primary function of keratinocytes is to form a physical barrier. Keratinocytes are also armed with an arsenal of danger-sensing receptors, including pathogen recognition receptors TLR1-6 and TLR9 (1) and $\mathrm{Ca}^{2+}$ channels that detect perturbations in temperature, pressure, and osmotic regulation $(2,3)$. Upon activation, keratinocytes initiate immune responses, releasing antimicrobial peptides ( $\beta$-defensins, REG3A, S100A7, and S100A8; ref. 4); cytokines (IL-6, TNF, IL-1 $\alpha$, IL-33, IL-36, and thymic stromal lymphopoietin [TSLP]; refs. 5, 6); and alarmins (high-mobility group protein box 1 and ATP; refs. 7, 8). Human, but not mouse keratinocytes, equipped with the NLRP1, NLRP3, and AIM2 inflammasomes, also cleave pro-IL-1 $\beta$ and pro-IL-18 into their active forms (9). Collectively, keratinocyte-derived cytokines initiate the sensations of itch and pain (10-13) and shape the outcome of immune responses by affecting the activation and migration of skin-resident immune cells. 
In the steady state, Langerhans cells are physically tethered to keratinocytes above the basal layer in the stratum spinosum. Epidermal retention of Langerhans cells requires TGF- $\beta 1$ signaling (14). Latent TGF- $\beta 1$ expressed on Langerhans cells is cleaved by keratinocyte-expressed integrins $\alpha_{\mathrm{v}} \beta_{6}$ or $\alpha_{\mathrm{v}} \beta_{8}(15)$, and keratinocyte-specific depletion of either $\alpha_{\mathrm{v}} \beta_{6}$ or $\alpha_{\mathrm{v}} \beta_{8}$ results in the loss of Langerhans cells in the epidermis (ref. 15 and Figure 1).

Langerhans cell maturation is promoted by pathogen-associated molecular patterns $(16,17)$, fragments of the ECM protein hyaluronan, as well as endogenous alarmins and cytokines produced by nearby keratinocytes $(5,18-20)$. Following activation, Langerhans cells extend dendrites through the tight junctions formed by keratinocytes in the stratum granulosum to acquire antigen. Langerhans cells, expressing the tight junction proteins claudin-1 and zonula occludens-1, form new tight junctions with keratinocytes (21). This mechanism allows Langerhans cells to sample antigen throughout the epidermis while maintaining keratinocyte barrier integrity.

Activation-induced maturation causes epidermal Langerhans cells to migrate toward skin-draining lymph nodes (reviewed extensively in refs. 22, 23). Migration of Langerhans cells is a multistep process involving sequential upregulation of CXCR4 and CCR7 chemokine receptors (24). Migration from the epidermis appears to depend on the EpCAM, which mediates cell-cell contact via the tight junction protein claudin-7, a variant of CD44, or E-cadherin expressed on keratinocytes (ref. 25 and Figure 1). Langerhans cell-specific ablation of EpCAM increases Langerhans cell migration to skin-draining lymph nodes following topical application of the contact sensitizer 2,4,6-trinitrochlorobenzene, though this effect may be context dependent $(26,27)$. Increased CXCR4 expression promotes Langerhans cell migration from the epidermis toward CXCL12 secreted by dermal fibroblasts (24). As Langerhans cells arrive in the dermis and continue to mature, upregulation of CCR7 allows them to respond to CCL19 and CCL21 released by lymphatic endothelial cells and migrate through the lymphatics to draining lymph nodes (28). Immature Langerhans cells may also migrate to the dermis in response to CCL2 and CCL5 secreted by dermal fibroblasts triggered by chemical irritants (29). However, lack of CCR7 expression by immature Langerhans cells prevents subsequent migration to draining lymph nodes (29). Migration of DCs from the skin also depends on controlled ECM degradation by MMP-9, MMP-2, and hyaluronidase expressed by DCs, keratinocytes, and other cells in the dermis, such as fibroblasts (30-32).

A major function of Langerhans cells is the delivery of processed antigen acquired in the skin to $\mathrm{T}$ cells in the lymph node to initiate adaptive immune responses. The precise function of Langerhans cells in the development of adaptive immunity, however, is not fully understood. The function of Langerhans cells has been best interrogated using mouse models in which Langerhans cells are depleted (recently reviewed elsewhere in ref. 33). For the contact hypersensitivity (CHS) model of allergic contact dermatitis, Langerhans cells are required only in the setting of very limited antigen density (14, 34-37). The bulk of the response is generated by cutaneous DC1s (cDC1s, classified in Table 1). During a Candida albicans infection, Langerhans cells are required for the development of protective Th17 responses and to reactivate skin-resident Th17 and Treg cells $(38,39)$.

\section{In the dermis: dermal DC and mast cells}

Within the epidermis, regulation of Langerhans cell-keratinocyte junctions permits Langerhans cells to carry out their primary functions of antigen presentation and promoting $\mathrm{T}$ cell responses. Within the dermis, the primary functions of innate immune cells are more diversified. In the steady state, the ECM-rich dermis houses mast cells, innate lymphoid cells (ILCs), heterogeneous dermal DC populations, and migratory Langerhans cells $(24,29)$. Contact-dependent and contact-independent communication among dermal DCs, dermal mast cells, and other cells in the surrounding microenvironment affect complex immune processes, including antigen delivery to innate effector cells, antigen presentation to T cells, and activation of neural circuits.

Dermal DCs. The primary function of dermal DCs, like that of their epidermal counterpart, Langerhans cells, is to deliver antigen to T cells. Several types of DCs have been identified within the skin and draining lymph nodes, and details regarding the phenotype, localization, and functional specialization of different DC subsets in mice and humans have been reviewed extensively $(23,33,34,40-42)$. Conventional DCs are typically located more superficially than dermal macrophages, which reside predominantly in deeper perivascular regions of the dermis $(43,44)$. Like Langerhans cells, dermal DCs acquire antigen within the skin and can present antigen both locally and in the skin-draining lymph nodes. Dermal DCs are grouped into multiple phenotypic categories with overlapping and distinct functions: $\mathrm{CDC} 1 \mathrm{~s}, \mathrm{cDC} 2 \mathrm{~s}$, DN DCs, and monocyte-derived DCs (ref. 23 and Table 1). The highest proportion of CD11b dermal DCs express CD301b (45). 


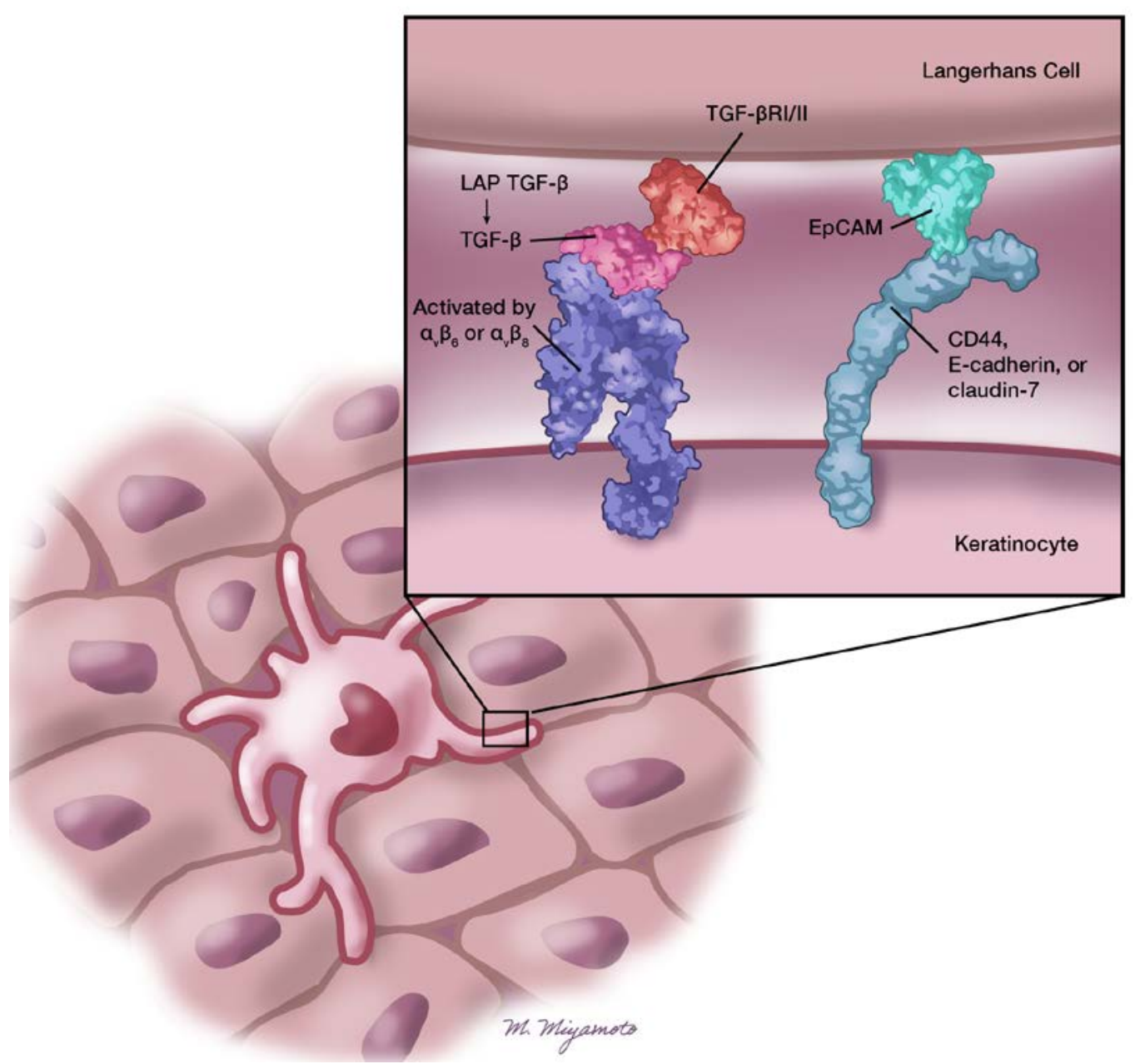

Figure 1. Langerhans cells interact with keratinocytes in the epidermis through multiple junctions. In the steady state, the retention of Langerhans cells in the epidermis requires the conversion of TGF- $\beta$ bound to the latency-associated peptide (LAP) to active TCF- $\beta$ by integrins $\alpha_{v} \beta_{6}$ or $\alpha_{v} \beta_{8}$ expressed on the keratinocyte surface. Interactions between the epithelial cell adhesion molecule (EpCAM) on Langerhans cells and claudin-7, a variant of CD44, or epithelial cadherin (E-cadherin) expressed on keratinocytes may regulate Langerhans cell migration. DLN: draining lymph node. Illustrated by Mao Miyamoto.

Genetic ablation strategies have been used to dissect the precise functions of dermal DC subsets in initiating adaptive immune responses. Transient depletion of $\mathrm{CD} 301 \mathrm{~b}^{+}$dermal DCs reduces Th2 responses to the parasite Nippostrongylus brasiliensis or to protein antigens delivered with Th2-polarizing adjuvants (4547) and reduces IL-17 responses in murine models of either psoriasiform dermatitis or Streptococcus pyogenes intranasal infection $(48,49)$. Studies evaluating mice constitutively or transiently deficient in the chemokine receptor XCR1 provide good insight into the general functions of dermal DCs in skin, although XCR1 is expressed in DCs in secondary lymphoid tissues and other peripheral tissues. Notably, inducible transient depletion of $\mathrm{XCR}^{+} \mathrm{DCs}$ impairs $\mathrm{CD}^{+} \mathrm{T}$ cell priming (50) and memory $\mathrm{CD} 8^{+} \mathrm{T}$ cell recall responses to secondary infection by intracellular pathogens (51). Mice constitutively lacking XCR $1^{+} \mathrm{DCs}$ have reduced numbers of intestinal T cells, which is attributed to diminished survival signals from XCR1 ${ }^{+} \mathrm{DCs}$ (52).

Although conventional dermal DCs efficiently activate $\mathrm{T}$ cells in both the skin and draining lymph nodes, monocyte-derived dermal DCs, which have inferior migratory ability and somewhat reduced $\mathrm{T}$ cell stimulatory capacity, are still able to activate tissue-resident memory $\mathrm{T}$ cells, Tregs, or recruited effectors in the skin (53). Under various conditions (e.g., infection, allergen exposure), dermal DCs may recruit tis- 
Table 1. Phenotypic classifications of cutaneous DCs in mice and the relationship with human cutaneous DC subsets

\begin{tabular}{|c|c|c|c|c|}
\hline Murine DC type & Primary location & Phenotypic markers & Primary functions & Human correlate \\
\hline Langerhans cells & Epidermis & $\begin{array}{c}\text { CD11b }^{+} \\
\text {CD207 }^{+} \\
\text {CD301b }^{-/+} \\
\text {EpCAM }^{+}\end{array}$ & $\begin{array}{l}\text { Tolerogenic immune responses; } \\
\text { promotes Th17 }\end{array}$ & Langerhans cells \\
\hline cDC1 & Dermis & $\begin{array}{c}\mathrm{CD}_{11 b^{-}} \\
\mathrm{CD}^{-} 03^{+} \\
\mathrm{XCR}^{+} \\
\mathrm{CD}^{+} \mathrm{C}^{+}\end{array}$ & $\begin{array}{l}\text { Cross priming, Th1 immune } \\
\text { responses }\end{array}$ & CD141+ DC \\
\hline DN DC & Dermis & 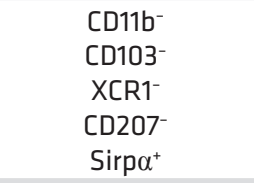 & Promotes Th2 immune responses & Unknown \\
\hline Monocyte-derived DC & Dermis & 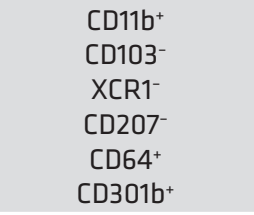 & $\begin{array}{l}\text { Local antigen presentation in skin; } \\
\text { formation of immune clusters }\end{array}$ & CD14 $^{+}$dermal cells \\
\hline
\end{tabular}

Adapted from ref. 35. CDC, conventional DC; DN DC, double-negative DC.

sue-resident memory $\mathrm{T}$ cells from within the skin or central memory $\mathrm{T}$ cells from circulation by secreting chemokines, including CCL5, CCL18, CCL20, and CCL22 (54-58). In response to allergen challenge, dermal macrophages produce CXCL2 to recruit DCs and T cells to form clusters in perivascular regions of the skin (59). In all cases, the resulting clusters of DCs and T cells facilitate DC-mediated activation and proliferation of antigen-specific $\mathrm{T}$ cells in the skin.

In addition to locally activating $T$ cells within the skin, dermal DCs carry acquired antigen to skin-draining lymph nodes, which primes naive and memory $\mathrm{T}$ cell responses. Following antigen uptake, CCR7 upregulation facilitates dermal DC migration to the draining lymph nodes $(28,60)$ in a manner similar to that described for Langerhans cells. Like Langerhans cells, dermal DCs enzymatically cleave a conduit through the ECM to reach the lymphatics, a process that appears to depend, in part, on interactions with dermal fibroblasts. In vitro coculture experiments show that ICAM-1 and Thy-1 expressed on cutaneous fibroblasts associate with $\beta_{2}$ integrin expressed on monocyte-derived DCs (61). During prolonged contact maintained by these cellular adhesion receptors, fibroblasts provide maturation cues to dermal DCs (62, 63). In particular, TNF-treated or IL- $\beta$-treated dermal fibroblasts induce expression of MMP9 by dermal DCs (61). Studies investigating the relationship between fibroblasts and dermal DCs in the context of nickel allergy demonstrate that nickel causes dermal fibroblasts to secrete IL-1 $\beta$ and CCL20, which promote DC maturation and recruitment of $\mathrm{CD} 8^{+} \mathrm{T}$ cells for cross-priming, respectively (64). Collectively, these interactions between dermal fibroblasts and DCs may remodel the ECM, allowing dermal DCs (and Langerhans cells) to travel through the dermis to the lymphatics to initiate $\mathrm{T}$ cell responses in skin-draining lymph nodes, as well as supporting local $\mathrm{T}$ cell activation in the skin.

Mast cells. Mast cell functions are diverse, including remodeling of tissue and activation of neighboring cells through the release of inflammatory mediators. As such, the distance between mast cells and their neighbors dictates the magnitude of the effects of mast cell activation. Like DCs, cutaneous mast cells are found in the superficial dermis near blood vessels (44) and nerves (65). Mast cells are stimulated to secrete proinflammatory and Th2 cytokines, as well as amphiregulin (66) by bacterial and viral ligands (67); cytokines, including IL-9 (68), IL-33 (69), and TSLP (70); and neuropeptides (65), as well as IgE. Canonical mast cell activation initiated via the IgE/FceRI pathway is well defined (reviewed in ref. 71). Perivascular 
mast cells extend processes through the vascular endothelium to sample the blood for IgE (72). Interactions between antigen-bound IgE and the FceRI trigger immediate release of protease- and histamine-rich granules and heparin-stabilized cytokines (73). The protease content of mast cell granules differs in mice and humans. Murine granules contain chymases (mast cell protease [MCP] 4 and MCP5), tryptases (MCP6 and MCP7), and carboxypeptidase A3 (CPA3), while human connective tissue-type mast cell granules contain $\alpha / \beta 1$ and $\beta 2 / \beta 3$ tryptase, chymase, and CPA3 (74). IgE also initiates de novo transcription of chemokines and cytokines, including TNF, IL-1 $\beta$, IL-4, IL-9, and IL-13 and synthesis of lipid mediators, such as prostaglandin (PG) $\mathrm{D}_{2}$ and $\mathrm{PGE}_{2}(75)$. Released later than granules, these inflammatory mediators extend the duration of mast cell influence in the microenvironment.

Mast cells are dynamic and have disparate functions in the skin. Releasing high levels of IL-4 and IL-13, mast cells are associated with atopy and Th2-skewed diseases $(76,77)$. Mast cells can also participate in Th17 responses $(77,78)$. In Th1-skewed immune responses, particularly in the murine models of CHS, mast cells have been shown to both exacerbate and mitigate immune responses (79-81). To resolve this discrepancy, mast cell functions in CHS were recently reevaluated using multiple lines of mast cell-deficient mice and different protocols to induce CHS (82). Mast cells suppressed the immune response in a chronic model of CHS whereas they were proinflammatory and increased immune sensitization using an acute CHS model (82). Surprisingly, in chronic CHS, $40 \%$ of mast cells transcribed antiinflammatory cytokine IL-10 following sensitization, and specifically depleting IL-10 in mast cells enhanced CHS (82). This important study resolved the seemingly conflicting results investigating the roles of mast cells in CHS, demonstrating that the roles of mast cells in complex cutaneous immune responses may differ based on the intensity of the reaction.

Mast cells are positioned to interact closely with fibroblasts. In a murine fibrosis model, heterotypic ICAM1 /integrin interactions tether fibroblasts to mast cells. In this model, keratinocyte-derived plasminogen activator inhibitor 1 enhances mast cell accumulation in the tissue and increases mast cell-fibroblast contact. Mast cells provide IL-4, IL-13, and fibroblast growth factors that promote fibroblast proliferation (83, 84). Mast cell-derived histamine initiates collagen production from dermal fibroblasts (85). While mast cells stimulate fibroblasts in the skin, dermal fibroblasts secrete retinoic acid-degrading enzymes, which block expression of the P2RX7 (a receptor for ATP) by mast cells, impeding ATP-initiated mast cell activation (86). Thus, in some circumstances, interactions between dermal fibroblasts and mast cells have a net effect of homeostatic maintenance.

Mast cells have also been identified in apposition to rare tissue-resident ILC2s in human and mouse skin (87). ILC2s are functionally similar to mast cells, with both cell types secreting IL-4, IL-5, IL-13, and amphiregulin following receptor-specific activation. Coordinated activation of mast cells and ILC2s may amplify protective antihelminth or damaging allergic inflammation. In the skin, ILC2s and mast cells physically associate through an as-yet-uncharacterized mechanism (87). ILC2s that are adjacent to mast cells are positioned to receive mast cell-derived activation signals. Human and mouse ILC2s are activated by IL-25, TSLP, and IL-33, which are released primarily by keratinocytes (88-90) but also by mast cells (69). In helminth infection in the gut, both mast cells and ILC2s accumulate, and IL-33, originating from ATP-stimulated mast cells, augments ILC2 activation (91). In the skin, where expression of P2RX7 is suppressed on mast cells (86), other mechanisms may regulate the ILC2/mast cell axis. For example, mast cell granules containing chymase and tryptase promote maturation of IL-33 (produced by mast cells or keratinocytes) into biologically active forms with enhanced potency. Compared with full-length IL-33, protease "edited" IL-33 more robustly expands ILC2 populations in vivo and activates ILC2s ex vivo (92). Through such mechanisms, the effect of mast cells on ILC2 activation and the production of type 2 cytokines may be additive and perpetuate inflammation in the skin.

\section{Interactions between mast cells and DCs in the skin}

Across multiple tissues from human and mice, mast cells directly interact with dermal DCs (93). IgE/antigen-activated mast cells maintain long-lasting contact with DCs via lymphocyte function-associated antigen 1 (LFA-1) and very late antigen 4 integrins (93). Contact between mast cells and DCs causes cytoplasmic reorientation, directing mast cell granule and cytokine release toward DCs and allowing transfer of IgE-bound antigens from mast cells to DCs (Figure 2). Mast cell/DC interactions alter local cytokine and chemokine production differentially depending on whether direct contact occurs (93). For example, in a contact-independent manner, mast cells cause DCs to reduce CCL2 and CCL3 secretion. Direct contact with mast cells prevents or delays this reduction. Coculture of bone marrow-derived DCs with peritoneal mast cells, but not mast cell-conditioned media, increased IFN- $\gamma$ and IL-6 release, suggesting a contact-dependent effect (79). Collectively, these studies highlight the involvement of both paracrine and contact-mediated signaling between these cells. 
Notably, DCs modulate levels of membrane-bound TNF on the surface of mast cells. TNF, in turn, matures DC in a contact-dependent manner (81). Similarly, peritoneal-derived mast cells mature DCs in vitro (79). In the skin, mast cell-derived TNF has been shown to induce the migration of nearby dermal DCs to the skin-draining lymph nodes to prime T cells in models of allergic and irritant CHS $(81,94)$. Mast cell-derived TNF also modulates DC populations distal to the skin. Selective depletion of TNF in cutaneous mast cells diminishes the capacity of lymphoid resident $\left(\mathrm{CD}^{+}\right)$DCs to prime $\mathrm{T}$ cells in the skin-draining lymph nodes (95). Through a mast cell intermediate, dermal DCs may regulate their own migratory path.

Soluble mast cell-derived factors are also involved in activation-induced migration of Langerhans cells. Langerhans cell migration, like that of dermal DCs, is induced by TNF and IL-1 $\beta(96,97)$. Langerhans cell migration in response to TLR2 or TLR7 ligands is dependent on TNF released from dermal mast cells (98, 99). In the course of allergic inflammation, other factors released by mast cells, including histamine, may direct Langerhans cell migration. Both human and mouse Langerhans cells express functional histamine receptors (100). In mouse models, FceRI signaling in mast cells drives Langerhans cell migration to the skin-draining lymph node in a histamine-dependent manner (101).

\section{Antigen transfer and adaptive immune responses}

In conventional models of DC function, antigen is acquired directly from pathogens in the peripheral tissue; however, there are many examples in which DCs obtain antigen through less conventional means. DCs may deputize innate immune cells, stromal cells, or other DCs for professional antigen presentation via transfer of antigen-MHC II complexes. Dermal DCs (102, 103), lymph node-resident DCs (104-107), or lymph node stromal cells (108) can receive antigen-MHC II complexes from migrating Langerhans cells and dermal DCs. For example, Langerhans cells infected by herpes simplex virus in the epidermis migrate to the dermis, where they form clusters with dermal DCs, undergo apoptosis, and are phagocytosed by the dermal DCs, which then migrate to draining lymph nodes (102). Similarly, migratory DCs can transfer antigen to more abundant lymph node-resident DCs (104-107).

A recent study demonstrated that cross-presentation of antigen from dying DCs and direct contact between DCs were not essential for antigen transfer but that LFA-1 expression on recipient DCs was required (104). Because LFA-1 is a scavenging receptor for DC-derived exosomes, exosome-mediated transfer of antigen is likely a predominant mechanism of antigen transfer between these DC populations (104). Antigen transfer from skin DCs enhances adaptive immune responses by disseminating antigen from a broader repertoire of DCs and by transfer of antigen from virally infected DCs with compromised antigen-processing capacity to uninfected DCs.

Transfer of antigen-MHC II complexes from DCs to mast cells endows mast cells with antigen-presenting function. Recent studies have demonstrated direct transfer of MHC II from migratory DCs to mast cells (Figure 1), as well as to basophils in the dermis $(109,110)$. This process of MHC II-antigen transfer from DCs to mast cells occurs directly via immunological synapse-like contacts, a process known as trogocytosis. During skin inflammation (e.g., following topical application of hapten), mobile DCs form immune synapse-like contacts with stationary mast cells in the dermis, leading to transfer of plasma membrane (containing MHC II) from DCs to mast cells (110). These "cross-dressed" mast cells induce $\mathrm{T}$ cell proliferation and skew T cells toward the Th1 pathway (109). Through some combination of these contact-independent mechanisms and contact-dependent trogocytosis, skin-resident mast cells affect $\mathrm{T}$ cell responses in the skin-draining lymph nodes through a DC intermediate.

Mast cells may initiate T cell activation using DC-acquired MHC II or with endogenous MHC II. Classical studies have shown that IFN- $\gamma$ stimulation increases MHC II on the surface of immortalized human mast cells (111) and on human mast cells differentiated from peripheral blood (112). Moreover, it has recently been shown that IFN- $\gamma$-stimulated human skin-derived mast cells increase MHC II expression, acquire and process antigen, and induce $\mathrm{T}$ cell responses ex vivo (113). In humans, the exact location of mast cell/T cell interactions is unknown. In mice, mast cell migration to the skin-draining lymph nodes has been observed in a CHS model (114) and following ultraviolet irradiation (115). Mast cell function diverges in these models; in CHS, mast cells promote local inflammation and may promote $\mathrm{T}$ cell-mediated immunity in the lymph nodes. In ultraviolet radiation models, mast cells in the lymph node interact with $\mathrm{B}$ cells and diminish $\mathrm{T}$ cell responses. Collectively, these studies suggest that mast cells may act as antigen-presenting cells in the skin-draining lymph nodes. 
MC-derived TNF enhances

CD8 $a^{+}$DC priming of $T$ cells in the skin DLN
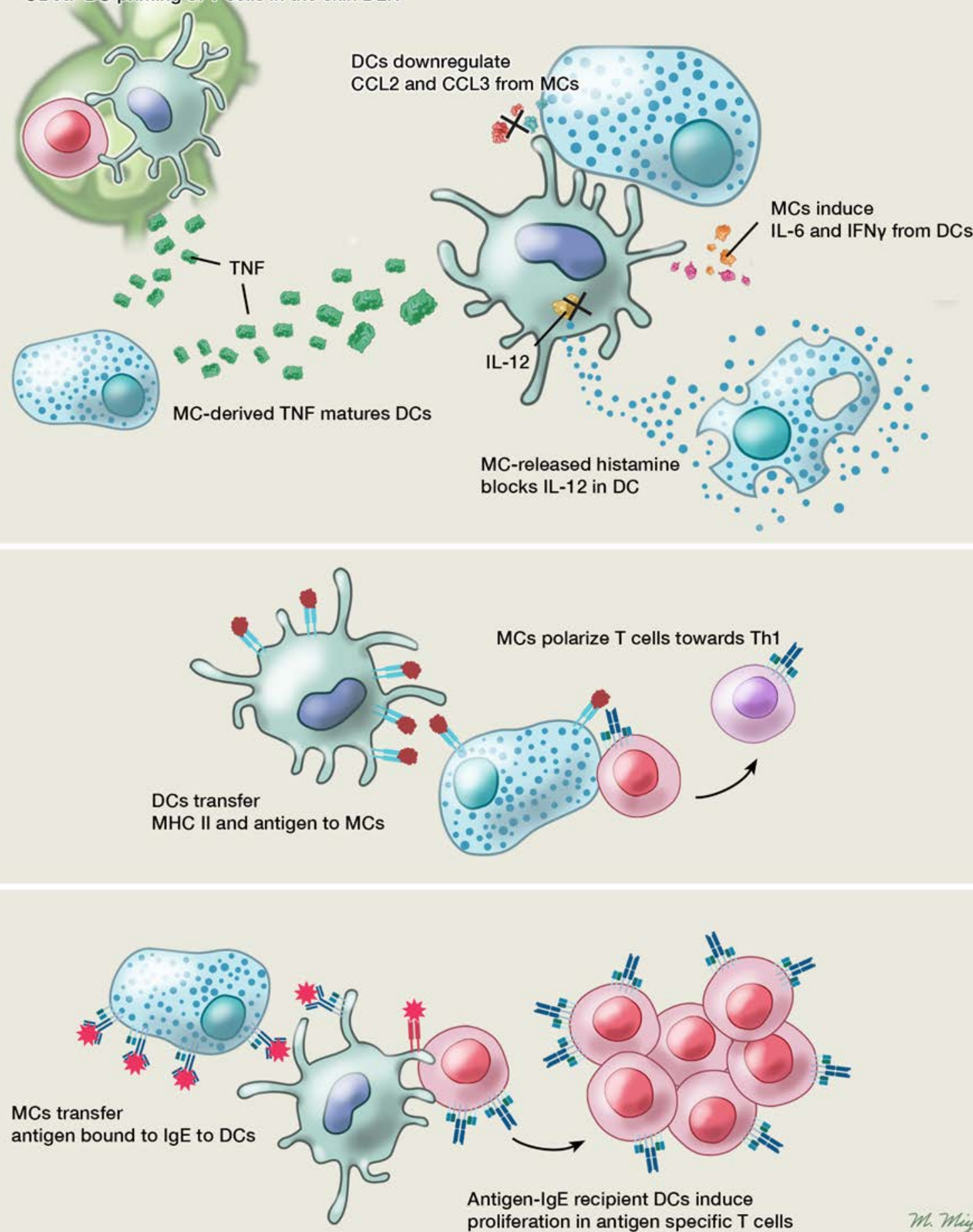

M. Migamoto

Figure 2. Dendritic cell-mast cell communication facilitates induction of adaptive immune responses. Contact-dependent and -independent mechanisms between DCs and mast cells (MCs) promote T cell responses. Illustrated by Mao Miyamoto.

Mast cells also reportedly transfer endocytosed antigen-IgE-FceR1 complexes to DCs through the synapse-like contact (93). DC recipients of mast cell-antigen-IgE complexes provide antigen-specific stimulation to $\mathrm{T}$ cells, resulting in $\mathrm{T}$ cell proliferation (93). The direct effects of antigen-IgE transfer from mast cells on the T cell-polarizing capacity of DCs were not evaluated in this particular study; however, other studies 
investigating the effects of "mast cell conditioning" on DCs have shown conflicting results. In vitro, mast cell-released histamine inhibits IL-12 expression in human DCs, blocking subsequent Th1 polarization and licensing the DC to polarize T cells toward a Th2 response $(116,117)$. However, DCs conditioned with mast cells have also been shown to skew T cells toward Th1 and Th17 phenotypes in vitro and in murine models of CHS and leishmaniasis $(79,81)$. The discrepancies in the reported outcomes of mast cell-DC communication on $\mathrm{T}$ cell skewing may reflect context-dependent differences in activation stimuli and in mouse models.

\section{Interactions with peripheral sensory nerve fibers}

Sensory afferent neurons modulate the functions of DCs and mast cells in antigen presentation and induction of innate inflammation. Myelinated nerve bundles, supported by Schwann cells, traverse vertically through the dermis (reviewed in refs. 118, 119). The nerve bundles lose their protective Schwann cells and terminate as either thinly myelinated A $\delta$ or unmyelinated C-fibers in either the dermis or the epidermis. Nerve fibers react to physical or chemical stimuli, releasing neuropeptides and cytokines that modify the cutaneous microenvironment (65).

Seminal work from Chui et al. $(120,121)$ demonstrated that sensory neurons are equipped to directly respond to bacterial infections in the skin and can serve as innate immune sensors. In addition, cutaneous afferents express receptors for many cytokines, such as TSLP, IL-4, IL-31, and IL-33 (11, 12, 122, 123). Thus, sensory afferents are able to directly detect pathogens and local inflammation-induced mediators, resulting in the transduction of pain and itch sensations. Keratinocyte-mediated nociceptive responses have been directly observed through optogenetic activation of keratinocytes engineered to respond to light, thereby resulting in activation of selective subsets of sensory neurons (13). Keratinocytes and other cellular sources of cytokines (mast cells, DCs, ILC2s) activate cutaneous afferents resulting in neuropeptide release into the microenvironment.

Both Langerhans cells and dermal DCs associate with nerve fibers in the skin $(124,125)$. Depletion of Langerhans cells reduces the density of epidermal nerve fibers (126), demonstrating the relationship between these cell types. This relationship is not unidirectional because neuropeptides communicate with Langerhans cells to alter induction of Th1, Th2, and Th17 responses (127-129).

Dermal DCs are adjacent to sensory fibers in the skin (125). In a recent study using the imiquimod model of psoriasis, blocking the activity of sensory nerve fibers inhibited IL-23 induction in dermal DCs and IL-17 production from dermal $\gamma \delta \mathrm{T}$ cells (125). Building on this finding, Kashem et al. (38) specifically linked release of the neuropeptide calcitonin gene-related peptide (CGRP) from $C$. albicans-sensing TRPV1-expressing cutaneous afferents to the IL-23 release from CD301b dermal DCs. Induction of IL-23, in turn, induced dermal $\gamma \delta \mathrm{T}$ cells to secrete IL-17A to control infection (38). Collectively, sensory nerves orchestrate local innate immune responses in the skin by modulating DC function.

Mast cells form a physical synapse with unmyelinated C-fibers and are exquisitely poised for bidirectional communication with neurons in the skin (130-133). Mast cell-derived TNF and nerve growth factor (NGF) stimulate neuronal elongation (74, 134); mast cell-derived histamine interacts with pruriceptors on C-fibers to induce itch and substance P release (10). Although in vitro studies suggest that CGRP has no effect on mast cells, classic in vivo studies using the 2,4-dinitrofluorobenezene CHS model suggest that CGRP inhibits mast cell function $(135,136)$. Another neuropeptide, substance $P$, augments mast cell effector functions, acting through either the neurokinin-1 receptor or the members of the Mas-related GPCR family to amplify the mast cell response to IgE (137-139). Substance P synergizes with IL-33 to activate mast cells, increasing VEGF release and TNF synthesis $(140,141)$. Substance P alone induces mast cell degranulation $(138,142,143)$. Interestingly, human mast cells stimulated with substance $\mathrm{P}$ alone release only $\mathrm{PGD}_{2}, \mathrm{PGE}_{2}$, and VEGF but not proinflammatory cytokines, such as TNF and IL-13. Generally, substance P induces a less robust mast cell response when compared with that initiated by $\operatorname{IgE}$ (138). Selective release of cytokines may limit immune responses and also cytokine-initiated neuronal sensations of itch or pain.

How nerves contact innate immune cells in the skin is insufficiently studied. Sensory nerve fibers are equipped with multiple immune adhesive molecules. In culture, dorsal root ganglion cells express ICAM-1 and vascular cellular adhesion molecule 1 when activated with NGF (144). Notably, interactions between the immunoglobulin-like adhesion molecule nectin-3, which is expressed on neurons, and synaptic cellular adhesion molecule (SynCAM/Cadm1) expressed on a subset of mast cells have been reported (130, 131). Nectin-3/SynCAM interactions facilitate the close relationship between mast cells and neurons. At the RNA level, expression of Cadm1 is higher in cutaneous mast cells, compared with the expression of those isolated from the oral mucosa (tongue), and Cadm1 expression is higher than other cellular adhesion 
molecule family members and higher than FceRla expression in skin mast cells (145), emphasizing the importance of this molecule in mast cell function. Though SynCAM expression has not been reported on cutaneous DCs, Langerhans cells also express Cadm1 mRNA, albeit at levels lower than that for Epcam and the TGF- $\beta$ receptor subunits. These data suggest that Langerhans cells may be equipped with the molecules necessary to form a close physical association with sensory nerve fibers. Ultimately, adhesive junctions formed among DCs, mast cells, and sensory neurons may facilitate neuroimmune communication and, subsequently, alter the generation of protective innate immune responses in the skin.

\section{Conclusions}

There are many possibilities for future directions of research into cutaneous immune responses, along with some cautions to consider. A critical barrier in cutaneous research at this time is the lack of concordance between mouse skin- and human skin-bioinformatic studies; comparing human and mouse shows approximately 30\% identity (146). Numerous differences between murine and human immunology (reviewed in ref. 147) emphasize the importance of translational studies to confirm that murine studies extend to humans. The integration of humanized mouse models into cutaneous immunology may resolve the human skin versus mouse skin issue. Bryce et al. (148) recently characterized mast cells in a humanized-mouse model. This type of model system provides a tool for manipulating human cells and their interactions in the skin.

Our understanding of cutaneous immune responses and intercellular communication can be advanced by extrapolating findings in other barrier tissues (e.g., gut and lung). Similarly, novel mechanisms identified in the skin could also be extended — with experimental validation - to mucosal tissues. Identifying common and divergent pathways in skin and mucosal tissues may lead to broader application of existing therapeutics or development of new ones that target specific barrier tissues.

Overall, it seems useful to view the complex series of interactions between innate immune cells in the skin as akin to molecular signaling pathways. Targeting upstream interactions may exert broad influence over downstream outcomes, while targeting downstream interactions may have more limited or specific effects. Just as mapping a molecular signaling pathway reveals potential targets for pharmacological intervention, understanding the complex series of cellular interactions in the skin provides insight into potential targets for manipulation to either enhance or suppress immune responses.

\section{Acknowledgments}

This manuscript was supported by National Institute of Arthritis and Musculoskeletal and Skin Diseases K01AR067250 to TLS, by National Cancer Institute T32CA175294 to SCB, and by National Institute of Arthritis and Musculoskeletal and Skin Diseases R01AR060744, R01AR067187, and R01AR071720 to DHK

Address correspondence to: Tina L. Sumpter, 200 Lothrop St, Ste W1155, Pittsburgh, Pennsylvania 15261, USA. Phone: 412.648.9972; Email: t1s44@pitt.edu. Or to: Daniel H. Kaplan, 200 Lothrop St, Ste W1043, Pittsburgh, Pennsylvania 15261, USA. Phone: 412.648.9471; Email: dankaplan@pitt.edu.

1. Hari A, Flach TL, Shi Y, Mydlarski PR. Toll-like receptors: role in dermatological disease. Mediators Inflamm. 2010;2010:437246.

2. Moqrich A, et al. Impaired thermosensation in mice lacking TRPV3, a heat and camphor sensor in the skin. Science. 2005;307(5714):1468-1472.

3. Müller M, et al. Specific TRPC6 channel activation, a novel approach to stimulate keratinocyte differentiation. J Biol Chem. 2008;283(49):33942-33954.

4. Yang D, Biragyn A, Kwak LW, Oppenheim JJ. Mammalian defensins in immunity: more than just microbicidal. Trends Immunol. 2002;23(6):291-296.

5. Gröne A. Keratinocytes and cytokines. Vet Immunol Immunopathol. 2002;88(1-2):1-12

6. Nedoszytko B, Sokołowska-Wojdyło M, Ruckemann-Dziurdzińska K, Roszkiewicz J, Nowicki RJ. Chemokines and cytokines network in the pathogenesis of the inflammatory skin diseases: atopic dermatitis, psoriasis and skin mastocytosis. Postepy Dermatol Alergol. 2014;31(2):84-91.

7. Straino S, et al. High-mobility group box 1 protein in human and murine skin: involvement in wound healing. J Invest Dermatol. 2008;128(6):1545-1553.

8. Burrell HE, et al. Human keratinocytes release ATP and utilize three mechanisms for nucleotide interconversion at the cell surface. J Biol Chem. 2005;280(33):29667-29676.

9. Sand J, et al. Expression of inflammasome proteins and inflammasome activation occurs in human, but not in murine keratinocytes. Cell Death Dis. 2018;9(2):24.

10. Green D, Dong X. The cell biology of acute itch. J Cell Biol. 2016;213(2):155-161. 
11. Wilson SR, et al. The epithelial cell-derived atopic dermatitis cytokine TSLP activates neurons to induce itch. Cell. 2013;155(2):285-295.

12. Liu B, et al. IL-33/ST2 signaling excites sensory neurons and mediates itch response in a mouse model of poison ivy contact allergy. Proc Natl Acad Sci U S A. 2016;113(47):E7572-E7579.

13. Baumbauer KM, et al. Keratinocytes can modulate and directly initiate nociceptive responses. Elife. 2015;4:e09674.

14. Kel JM, Girard-Madoux MJ, Reizis B, Clausen BE. TGF- $\beta$ is required to maintain the pool of immature Langerhans cells in the epidermis. J Immunol. 2010;185(6):3248-3255.

15. Mohammed J, et al. Stromal cells control the epithelial residence of DCs and memory T cells by regulated activation of TGF- $\beta$. Nat Immunol. 2016;17(4):414-421.

16. Reis e Sousa C. Activation of dendritic cells: translating innate into adaptive immunity. Curr Opin Immunol. 2004;16(1):21-25

17. Joffre O, Nolte MA, Spörri R, Reis e Sousa C. Inflammatory signals in dendritic cell activation and the induction of adaptive immunity. Immunol Rev. 2009;227(1):234-247.

18. Nestle FO, Di Meglio P, Qin JZ, Nickoloff BJ. Skin immune sentinels in health and disease. Nat Rev Immunol. 2009;9(10):679-691.

19. Martin SF, et al. Mechanisms of chemical-induced innate immunity in allergic contact dermatitis. Allergy. 2011;66(9):1152-1163.

20. Kaplan DH, Igyártó BZ, Gaspari AA. Early immune events in the induction of allergic contact dermatitis. Nat Rev Immunol. 2012;12(2):114-124

21. Kubo A, Nagao K, Yokouchi M, Sasaki H, Amagai M. External antigen uptake by Langerhans cells with reorganization of epidermal tight junction barriers. J Exp Med. 2009;206(13):2937-2946.

22. Igyártó BZ, Kaplan DH. Antigen presentation by Langerhans cells. Curr Opin Immunol. 2013;25(1):115-119.

23. Kashem SW, Haniffa M, Kaplan DH. Antigen-presenting cells in the skin. Annu Rev Immunol. 2017;35:469-499.

24. Ouwehand K, Santegoets SJ, Bruynzeel DP, Scheper RJ, de Gruij1 TD, Gibbs S. CXCL12 is essential for migration of activated Langerhans cells from epidermis to dermis. Eur J Immunol. 2008;38(11):3050-3059.

25. Trzpis M, McLaughlin PM, de Leij LM, Harmsen MC. Epithelial cell adhesion molecule: more than a carcinoma marker and adhesion molecule. Am J Pathol. 2007;171(2):386-395.

26. Gaiser MR, et al. Cancer-associated epithelial cell adhesion molecule (EpCAM; CD326) enables epidermal Langerhans cell motility and migration in vivo. Proc Natl Acad Sci U S A. 2012;109(15):E889-E897.

27. Ouchi T, Nakato G, Udey MC. EpCAM expressed by murine epidermal Langerhans cells modulates immunization to an epicutaneously applied protein antigen. J Invest Dermatol. 2016;136(8):1627-1635.

28. Ohl L, et al. CCR7 governs skin dendritic cell migration under inflammatory and steady-state conditions. Immunity 2004;21(2):279-288

29. Ouwehand K, Scheper RJ, de Gruij1 TD, Gibbs S. Epidermis-to-dermis migration of immature Langerhans cells upon topical irritant exposure is dependent on CCL2 and CCL5. Eur J Immunol. 2010;40(7):2026-2034.

30. Muto J, et al. Hyaluronan digestion controls DC migration from the skin. J Clin Invest. 2014;124(3):1309-1319.

31. Ratzinger G, et al. Matrix metalloproteinases 9 and 2 are necessary for the migration of Langerhans cells and dermal dendritic cells from human and murine skin. J Immunol. 2002;168(9):4361-4371.

32. Han YP, Tuan TL, Hughes M, Wu H, Garner WL. Transforming growth factor- $\beta$ - and tumor necrosis factor- $\alpha$-mediated induction and proteolytic activation of MMP-9 in human skin. J Biol Chem. 2001;276(25):22341-22350.

33. Kaplan DH. Ontogeny and function of murine epidermal Langerhans cells. Nat Immunol. 2017;18(10):1068-1075

34. Clausen BE, Stoitzner P. Functional specialization of skin dendritic cell subsets in regulating T cell responses. Front Immunol. 2015;6:534.

35. Kautz-Neu K, et al. Langerhans cells are negative regulators of the anti-Leishmania response. J Exp Med. 2011;208(5):885-891.

36. Nagao K, et al. Murine epidermal Langerhans cells and langerin-expressing dermal dendritic cells are unrelated and exhibit distinct functions. Proc Natl Acad Sci U S A. 2009;106(9):3312-3317.

37. Noordegraaf M, Flacher V, Stoitzner P, Clausen BE. Functional redundancy of Langerhans cells and Langerin ${ }^{+}$dermal dendritic cells in contact hypersensitivity. J Invest Dermatol. 2010;130(12):2752-2759.

38. Kashem SW, Riedl MS, Yao C, Honda CN, Vulchanova L, Kaplan DH. Nociceptive sensory fibers drive interleukin-23 production from $\mathrm{CD} 301 \mathrm{~b}^{+}$dermal dendritic cells and drive protective cutaneous immunity. Immunity. 2015;43(3):515-526.

39. Seneschal J, Clark RA, Gehad A, Baecher-Allan CM, Kupper TS. Human epidermal Langerhans cells maintain immune homeostasis in skin by activating skin resident regulatory T cells. Immunity. 2012;36(5):873-884.

40. Haniffa M, Gunawan M, Jardine L. Human skin dendritic cells in health and disease. J Dermatol Sci. 2015;77(2):85-92.

41. Malissen B, Tamoutounour S, Henri S. The origins and functions of dendritic cells and macrophages in the skin. Nat Rev Immunol. 2014;14(6):417-428.

42. Mildner A, Jung S. Development and function of dendritic cell subsets. Immunity. 2014;40(5):642-656.

43. Wang XN, et al. A three-dimensional atlas of human dermal leukocytes, lymphatics, and blood vessels. J Invest Dermatol. 2014;134(4):965-974.

44. Tong PL, et al. The skin immune atlas: three-dimensional analysis of cutaneous leukocyte subsets by multiphoton microscopy. $J$ Invest Dermatol. 2015;135(1):84-93.

45. Kumamoto Y, Denda-Nagai K, Aida S, Higashi N, Irimura T. MGL2 Dermal dendritic cells are sufficient to initiate contact hypersensitivity in vivo. PLoS One. 2009;4(5):e5619.

46. Kumamoto Y, Linehan M, Weinstein JS, Laidlaw BJ, Craft JE, Iwasaki A. CD301b ${ }^{+}$dermal dendritic cells drive T helper 2 cell-mediated immunity. Immunity. 2013;39(4):733-743.

47. Kumamoto Y, Hirai T, Wong PW, Kaplan DH, Iwasaki A. CD301b+ dendritic cells suppress T follicular helper cells and antibody responses to protein antigens. Elife. 2016;5:e17979.

48. Kim TG, et al. Skin-specific CD301b ${ }^{+}$dermal dendritic cells drive IL-17-mediated psoriasis-like immune response in mice. $J$ Invest Dermatol. 2018;138(4):844-853.

49. Linehan JL, Dileepan T, Kashem SW, Kaplan DH, Cleary P, Jenkins MK. Generation of Th17 cells in response to intranasal infection requires TGF- $\beta 1$ from dendritic cells and IL-6 from CD301b ${ }^{+}$dendritic cells. Proc Natl Acad Sci U S A. 2015;112(41):12782-12787. 
50. Yamazaki C, et al. Critical roles of a dendritic cell subset expressing a chemokine receptor, XCR1. J Immunol. 2013;190(12):6071-6082.

51. Alexandre YO, Ghilas S, Sanchez C, Le Bon A, Crozat K, Dalod M. XCR1 $1^{+}$dendritic cells promote memory CD8 ${ }^{+}$T cell recall upon secondary infections with Listeria monocytogenes or certain viruses. J Exp Med. 2016;213(1):75-92.

52. Ohta T, et al. Crucial roles of XCR1-expressing dendritic cells and the XCR1-XCL1 chemokine axis in intestinal immune homeostasis. Sci Rep. 2016;6:23505.

53. Tamoutounour $\mathrm{S}$, et al. Origins and functional specialization of macrophages and of conventional and monocyte-derived dendritic cells in mouse skin. Immunity. 2013;39(5):925-938.

54. Collins N, et al. Skin CD4(+) memory T cells exhibit combined cluster-mediated retention and equilibration with the circulation. Nat Commun. 2016;7:11514.

55. Günther C, et al. CCL18 is expressed in atopic dermatitis and mediates skin homing of human memory T cells. J Immunol. 2005;174(3):1723-1728.

56. Kim TG, et al. Dermal clusters of mature dendritic cells and T cells are associated with the CCL20/CCR6 chemokine system in chronic psoriasis. J Invest Dermatol. 2014;134(5):1462-1465.

57. Katou F, et al. Macrophage-derived chemokine (MDC/CCL22) and CCR4 are involved in the formation of T lymphocyte-dendritic cell clusters in human inflamed skin and secondary lymphoid tissue. Am J Pathol. 2001;158(4):1263-1270.

58. Pivarcsi A, et al. CC chemokine ligand 18, an atopic dermatitis-associated and dendritic cell-derived chemokine, is regulated by staphylococcal products and allergen exposure. J Immunol. 2004;173(9):5810-5817.

59. Natsuaki Y, et al. Perivascular leukocyte clusters are essential for efficient activation of effector T cells in the skin. Nat Immunol. 2014;15(11):1064-1069.

60. Randolph GJ, Angeli V, Swartz MA. Dendritic-cell trafficking to lymph nodes through lymphatic vessels. Nat Rev Immunol. 2005;5(8):617-628.

61. Saalbach A, et al. Dermal fibroblasts induce maturation of dendritic cells. J Immunol. 2007;178(8):4966-4974.

62. Vistejnova L, et al. Low molecular weight hyaluronan mediated CD44 dependent induction of IL-6 and chemokines in human dermal fibroblasts potentiates innate immune response. Cytokine. 2014;70(2):97-103.

63. Kolár M, et al. Upregulation of IL-6, IL-8 and CXCL-1 production in dermal fibroblasts by normal/malignant epithelial cells in vitro: immunohistochemical and transcriptomic analyses. Biol Cell. 2012;104(12):738-751.

64. Gölz L, et al. Differences in human gingival and dermal fibroblasts may contribute to oral-induced tolerance against nickel. $J$ Allergy Clin Immunol. 2016;138(4):1202-1205.e3.

65. Roosterman D, Goerge T, Schneider SW, Bunnett NW, Steinhoff M. Neuronal control of skin function: the skin as a neuroimmunoendocrine organ. Physiol Rev. 2006;86(4):1309-1379.

66. Zaiss DM, et al. Amphiregulin enhances regulatory $\mathrm{T}$ cell-suppressive function via the epidermal growth factor receptor. Immunity. 2013;38(2):275-284.

67. Sandig H, Bulfone-Paus S. TLR signaling in mast cells: common and unique features. Front Immunol. 2012;3:185.

68. Sismanopoulos N, et al. IL-9 induces VEGF secretion from human mast cells and IL-9/IL-9 receptor genes are overexpressed in atopic dermatitis. PLoS One. 2012;7(3):e33271.

69. Hsu CL, Neilsen CV, Bryce PJ. IL-33 is produced by mast cells and regulates IgE-dependent inflammation. PLoS One. 2010;5(8):e11944.

70. Han NR, et al. TSLP induces mast cell development and aggravates allergic reactions through the activation of MDM2 and STAT6. J Invest Dermatol. 2014;134(10):2521-2530.

71. Galli SJ, Tsai M. IgE and mast cells in allergic disease. Nat Med. 2012;18(5):693-704.

72. Cheng LE, Hartmann K, Roers A, Krummel MF, Locksley RM. Perivascular mast cells dynamically probe cutaneous blood vessels to capture immunoglobulin E. Immunity. 2013;38(1):166-175.

73. Kunder CA, et al. Mast cell-derived particles deliver peripheral signals to remote lymph nodes. J Exp Med. 2009;206(11):2455-2467.

74. Wernersson S, Pejler G. Mast cell secretory granules: armed for battle. Nat Rev Immunol. 2014;14(7):478-494.

75. Moon TC, Befus AD, Kulka M. Mast cell mediators: their differential release and the secretory pathways involved. Front Immunol. 2014;5:569.

76. Kawakami T, Ando T, Kimura M, Wilson BS, Kawakami Y. Mast cells in atopic dermatitis. Curr Opin Immunol. 2009;21(6):666-678

77. Mashiko S, Bouguermouh S, Rubio M, Baba N, Bissonnette R, Sarfati M. Human mast cells are major IL-22 producers in patients with psoriasis and atopic dermatitis. J Allergy Clin Immunol. 2015;136(2):351-9.e1.

78. Lin AM, et al. Mast cells and neutrophils release IL-17 through extracellular trap formation in psoriasis. J Immunol. 2011;187(1):490-500.

79. Dudeck A, Suender CA, Kostka SL, von Stebut E, Maurer M. Mast cells promote Th1 and Th17 responses by modulating dendritic cell maturation and function. Eur J Immunol. 2011;41(7):1883-1893.

80. Grimbaldeston MA, Nakae S, Kalesnikoff J, Tsai M, Galli SJ. Mast cell-derived interleukin 10 limits skin pathology in contact dermatitis and chronic irradiation with ultraviolet B. Nat Immunol. 2007;8(10):1095-1104.

81. Otsuka A, et al. Requirement of interaction between mast cells and skin dendritic cells to establish contact hypersensitivity. PLoS One. 2011;6(9):e25538.

82. Reber LL, et al. Imaging protective mast cells in living mice during severe contact hypersensitivity. JCI Insight. 2017;2(9):e92900

83. Pincha N, et al. PAI1 mediates fibroblast-mast cell interactions in skin fibrosis. J Clin Invest. 2018;128(5):1807-1819.

84. Artuc M, Steckelings UM, Henz BM. Mast cell-fibroblast interactions: human mast cells as source and inducers of fibroblast and epithelial growth factors. J Invest Dermatol. 2002;118(3):391-395.

85. Yang L, et al. Histamine contributes to tissue remodeling via periostin expression. J Invest Dermatol. 2014;134(8):2105-2113.

86. Kurashima Y, et al. Extracellular ATP mediates mast cell-dependent intestinal inflammation through P2X7 purinoceptors. Nat Commun. 2012;3:1034.

87. Roediger B, et al. Cutaneous immunosurveillance and regulation of inflammation by group 2 innate lymphoid cells. Nat Immunol. 2013;14(6):564-573.

88. Walker JA, Barlow JL, McKenzie AN. Innate lymphoid cells — how did we miss them? Nat Rev Immunol. 2013;13(2):75-87. 
89. Camelo A, et al. IL-33, IL-25, and TSLP induce a distinct phenotypic and activation profile in human type 2 innate lymphoid cells. Blood Adv. 2017;1(10):577-589.

90. Imai Y, et al. Skin-specific expression of IL-33 activates group 2 innate lymphoid cells and elicits atopic dermatitis-like inflammation in mice. Proc Natl Acad Sci U S A. 2013;110(34):13921-13926.

91. Shimokawa C, et al. Mast cells are crucial for induction of group 2 innate lymphoid cells and clearance of helminth infections. Immunity. 2017;46(5):863-874.e4.

92. Lefrançais E, et al. Central domain of IL-33 is cleaved by mast cell proteases for potent activation of group- 2 innate lymphoid cells. Proc Natl Acad Sci U S A. 2014;111(43):15502-15507.

93. Carroll-Portillo A, et al. Mast cells and dendritic cells form synapses that facilitate antigen transfer for $\mathrm{T}$ cell activation. $J$ Cell Biol. 2015;210(5):851-864

94. Suto H, Nakae S, Kakurai M, Sedgwick JD, Tsai M, Galli SJ. Mast cell-associated TNF promotes dendritic cell migration. $J$ Immunol. 2006;176(7):4102-4112.

95. Dudeck J, et al. Mast-cell-derived TNF amplifies CD8(+) dendritic cell functionality and CD8(+) T cell priming. Cell Rep. 2015;13(2):399-411.

96. Cumberbatch M, Bhushan M, Dearman RJ, Kimber I, Griffiths CE. IL-1 $\beta$-induced Langerhans' cell migration and TNF- $\alpha$ production in human skin: regulation by lactoferrin. Clin Exp Immunol. 2003;132(2):352-359.

97. Cumberbatch M, Griffiths CE, Tucker SC, Dearman RJ, Kimber I. Tumour necrosis factor- $\alpha$ induces Langerhans cell migration in humans. Br J Dermatol. 1999;141(2):192-200.

98. Heib V, et al. Mast cells are crucial for early inflammation, migration of Langerhans cells, and CTL responses following topical application of TLR7 ligand in mice. Blood. 2007;110(3):946-953.

99. Jawdat DM, Rowden G, Marshall JS. Mast cells have a pivotal role in TNF-independent lymph node hypertrophy and the mobilization of Langerhans cells in response to bacterial peptidoglycan. J Immunol. 2006;177(3):1755-1762.

100. Gschwandtner M, et al. Murine and human Langerhans cells express a functional histamine $\mathrm{H} 4$ receptor: modulation of cell migration and function. Allergy. 2010;65(7):840-849.

101. Jawdat DM, Albert EJ, Rowden G, Haidl ID, Marshall JS. IgE-mediated mast cell activation induces Langerhans cell migration in vivo. J Immunol. 2004;173(8):5275-5282.

102. Kim M, et al. Relay of herpes simplex virus between Langerhans cells and dermal dendritic cells in human skin. PLoS Pathog. 2015;11(4):e1004812.

103. Yao C, Kaplan DH. Langerhans cells transfer targeted antigen to dermal dendritic cells and acquire major histocompatibility complex II in vivo. J Invest Dermatol. 2018;138(7):1665-1668.

104. Gurevich I, et al. Active dissemination of cellular antigens by DCs facilitates CD8 $8^{+}$T-cell priming in lymph nodes. Eur J Immunol. 2017;47(10):1802-1818.

105. Mueller SN. Spreading the load: antigen transfer between migratory and lymph node-resident dendritic cells promotes T-cell priming. Eur J Immunol. 2017;47(10):1798-1801.

106. Smyth LA, et al. Acquisition of MHC:peptide complexes by dendritic cells contributes to the generation of antiviral CD8 ${ }^{+} \mathrm{T}$ cell immunity in vivo. J Immunol. 2012;189(5):2274-2282.

107. Allan RS, et al. Migratory dendritic cells transfer antigen to a lymph node-resident dendritic cell population for efficient CTL priming. Immunity. 2006;25(1):153-162.

108. Dubrot J, et al. Lymph node stromal cells acquire peptide-MHCII complexes from dendritic cells and induce antigen-specific CD4 ${ }^{+} \mathrm{T}$ cell tolerance. J Exp Med. 2014;211(6):1153-1166.

109. Dudeck J, et al. Mast cells acquire MHCII from dendritic cells during skin inflammation. J Exp Med. 2017;214(12):3791-3811.

110. Miyake K, Shiozawa N, Nagao T, Yoshikawa S, Yamanishi Y, Karasuyama H. Trogocytosis of peptide-MHC class II complexes from dendritic cells confers antigen-presenting ability on basophils. Proc Natl Acad Sci U S A. 2017;114(5):1111-1116.

111. Love KS, Lakshmanan RR, Butterfield JH, Fox CC. IFN-gamma-stimulated enhancement of MHC class II antigen expression by the human mast cell line HMC-1. Cell Immunol. 1996;170(1):85-90.

112. Suurmond J, et al. Communication between human mast cells and CD4(+) T cells through antigen-dependent interactions. Eur J Immunol. 2013;43(7):1758-1768.

113. Lotfi-Emran S, et al. Human mast cells present antigen to autologous CD4+ T cells. J Allergy Clin Immunol. 2018;141(1):311-321.e10.

114. Wang HW, Tedla N, Lloyd AR, Wakefield D, McNeil PH. Mast cell activation and migration to lymph nodes during induction of an immune response in mice. J Clin Invest. 1998;102(8):1617-1626.

115. Byrne SN, Limón-Flores AY, Ullrich SE. Mast cell migration from the skin to the draining lymph nodes upon ultraviolet irradiation represents a key step in the induction of immune suppression. J Immunol. 2008;180(7):4648-4655.

116. Mazzoni A, Young HA, Spitzer JH, Visintin A, Segal DM. Histamine regulates cytokine production in maturing dendritic cells, resulting in altered T cell polarization. J Clin Invest. 2001;108(12):1865-1873.

117. Caron G, et al. Histamine polarizes human dendritic cells into Th2 cell-promoting effector dendritic cells. J Immunol. 2001;167(7):3682-3686.

118. McGlone F, Spence C. The cutaneous senses: touch, temperature, pain/itch, and pleasure. Neurosci Biobehav Rev. 2010;34(2):145-147.

119. Myers MI, Peltier AC, Li J. Evaluating dermal myelinated nerve fibers in skin biopsy. Muscle Nerve. 2013;47(1):1-11.

120. Chiu IM, et al. Bacteria activate sensory neurons that modulate pain and inflammation. Nature. 2013;501(7465):52-57.

121. Pinho-Ribeiro FA, et al. Blocking neuronal signaling to immune cells treats streptococcal invasive infection. Cell. 2018;173(5):1083-1097.e22.

122. Meng J, et al. New mechanism underlying IL-31-induced atopic dermatitis. J Allergy Clin Immunol. 2018;141(5):1677-1689.e8.

123. Oetjen LK, et al. Sensory neurons co-opt classical immune signaling pathways to mediate chronic itch. Cell. 2017;171(1):217-228.e13.

124. Hosoi J, et al. Regulation of Langerhans cell function by nerves containing calcitonin gene-related peptide. Nature. 1993;363(6425):159-163.

125. Riol-Blanco L, et al. Nociceptive sensory neurons drive interleukin-23-mediated psoriasiform skin inflammation. Nature. 2014;510(7503):157-161. 
126. Doss AL, Smith PG. Langerhans cells regulate cutaneous innervation density and mechanical sensitivity in mouse footpad. Neurosci Lett. 2014;578:55-60.

127. Ding W, Stohl LL, Wagner JA, Granstein RD. Calcitonin gene-related peptide biases Langerhans cells toward Th2-type immunity. J Immunol. 2008;181(9):6020-6026.

128. Janelsins BM, et al. Proinflammatory tachykinins that signal through the neurokinin 1 receptor promote survival of dendritic cells and potent cellular immunity. Blood. 2009;113(13):3017-3026.

129. Janelsins BM, et al. Neurokinin-1 receptor agonists bias therapeutic dendritic cells to induce type 1 immunity by licensing host dendritic cells to produce IL-12. Blood. 2013;121(15):2923-2933.

130. Ito A, Oonuma J. Direct interaction between nerves and mast cells mediated by the SgIGSF/SynCAM adhesion molecule. $J$ Pharmacol Sci. 2006;102(1):1-5.

131. Furuno $\mathrm{T}$, et al. The spermatogenic Ig superfamily/synaptic cell adhesion molecule mast-cell adhesion molecule promotes interaction with nerves. J Immunol. 2005;174(11):6934-6942.

132.Kleij HP, Bienenstock J. Significance of conversation between mast cells and nerves. Allergy Asthma Clin Immunol. 2005;1(2):65-80.

133. Undem BJ, Taylor-Clark T. Mechanisms underlying the neuronal-based symptoms of allergy. J Allergy Clin Immunol. 2014;133(6):1521-1534

134. Kakurai M, Monteforte R, Suto H, Tsai M, Nakae S, Galli SJ. Mast cell-derived tumor necrosis factor can promote nerve fiber elongation in the skin during contact hypersensitivity in mice. Am J Pathol. 2006;169(5):1713-1721.

135. Niizeki H, Alard P, Streilein JW. Calcitonin gene-related peptide is necessary for ultraviolet B-impaired induction of contact hypersensitivity. J Immunol. 1997;159(11):5183-5186.

136. Kulka M, Sheen CH, Tancowny BP, Grammer LC, Schleimer RP. Neuropeptides activate human mast cell degranulation and chemokine production. Immunology. 2008;123(3):398-410.

137. Sumpter TL, et al. Autocrine hemokinin-1 functions as an endogenous adjuvant for IgE-mediated mast cell inflammatory responses. J Allergy Clin Immunol. 2015;135(4):1019-30.e8.

138. Gaudenzio N, et al. Different activation signals induce distinct mast cell degranulation strategies. J Clin Invest. 2016;126(10):3981-3998.

139. Yano H, Wershil BK, Arizono N, Galli SJ. Substance P-induced augmentation of cutaneous vascular permeability and granulocyte infiltration in mice is mast cell dependent. J Clin Invest. 1989;84(4):1276-1286.

140. Taracanova A, et al. SP and IL-33 together markedly enhance TNF synthesis and secretion from human mast cells mediated by the interaction of their receptors. Proc Natl Acad Sci U S A. 2017;114(20):E4002-E4009.

141. Theoharides TC, et al. IL-33 augments substance P-induced VEGF secretion from human mast cells and is increased in psoriatic skin. Proc Natl Acad Sci U S A. 2010;107(9):4448-4453.

142. Li WW, Guo TZ, Liang DY, Sun Y, Kingery WS, Clark JD. Substance P signaling controls mast cell activation, degranulation, and nociceptive sensitization in a rat fracture model of complex regional pain syndrome. Anesthesiology. 2012;116(4):882-895.

143. Matsuda H, Kawakita K, Kiso Y, Nakano T, Kitamura Y. Substance P induces granulocyte infiltration through degranulation of mast cells. J Immunol. 1989;142(3):927-931.

144. Foster EL, et al. Eosinophils increase neuron branching in human and murine skin and in vitro. PLoS One. 2011;6(7):e22029.

145. Heng TS, Painter MW, Immunological Genome Project Consortium. The Immunological Genome Project: networks of gene expression in immune cells. Nat Immunol. 2008;9(10):1091-1094.

146. Gerber PA, Buhren BA, Schrumpf H, Homey B, Zlotnik A, Hevezi P. The top skin-associated genes: a comparative analysis of human and mouse skin transcriptomes. Biol Chem. 2014;395(6):577-591

147. Mestas J, Hughes CC. Of mice and not men: differences between mouse and human immunology. J Immunol. 2004;172(5):2731-2738.

148. Bryce PJ, et al. Humanized mouse model of mast cell-mediated passive cutaneous anaphylaxis and passive systemic anaphylaxis. J Allergy Clin Immunol. 2016;138(3):769-779. 